\title{
Does cognitive rehabilitation improve occupational outcomes including employment and activities of daily living, as well as quality of life and community integration in individuals with traumatic brain injury? - A Cochrane Review summary with commentary
}

\section{Aydan Oral}

Department of Physical Medicine and Rehabilitation, Istanbul Faculty of Medicine, Istanbul University, Istanbul, Turkey

E-mail:aydanoral@yahoo.com

The aim of this commentary is to discuss in a rehabilitation perspective the published Cochrane Review "Cognitive rehabilitation for adults with traumatic brain injury to improve occupational outcomes" by Kumar, Samuelkamaleshkumar, Viswanathan, \& Macaden ${ }^{1}$, under the direct supervision of Cochrane Work. This Cochrane Corner is produced in agreement with NeuroRehabilitation by Cochrane Rehabilitation.

\footnotetext{
${ }^{1}$ The abstract/plain language summary of this Cochrane Review is taken from a Cochrane Review previously published in the Cochrane Database of Systematic Reviews 2017, Issue 6, DOI: 10.1002/14651858.CD7935 (see www.cochranelibrary.com for information). Cochrane Reviews are regularly updated as new evidence emerges and in response to feedback, and Cochrane Database of Systematic Reviews should be consulted for the most recent version of the review.
}

\section{Background}

Evidence suggests beneficial effects of various types of cognitive rehabilitation on impairments in cognitive functions such as memory problems, attention deficits, or deficits in executive functioning in persons with traumatic brain injury (TBI) (Cicerone et al., 2011). However, little is known about the effectiveness of cognitive rehabilitation on functional outcomes including activities and participation such as activities of daily living (ADL), work participation, and ultimately effective participation in society/community life for persons with TBI. Rehabilitation has also been called the "medicine of functioning" and is conceptually described based on the International Classification of Functioning, Disability and Health (ICF) (WHO, 2001). It addresses impairments in body functions, activity limitations, 
and participation restrictions for improving physical and cognitive functioning and participation in people experiencing disabilities (European Physical and Rehabilitation Medicine Bodies Alliance, 2018). Therefore, it would be of great importance for rehabilitation professionals to know the effectiveness of an intervention on specific functioning outcomes relevant to activities and participation component of the ICF. The significant question of whether cognitive rehabilitation can help in improving occupational outcomes including employment/return-to-work and ADL as well as quality of life (QoL) and community integration in individuals with TBI is specifically addressed by the below Cochrane Review:

\section{Cognitive rehabilitation for adults with trau- matic brain injury to improve occupational out- comes}

(Kumar, Samuelkamaleshkumar, Viswanathan, \& Macaden, 2017).

\section{What is the aim of the Cochrane Review?}

The aim of this Cochrane Review was to assess the effectiveness of cognitive rehabilitation on occupational activities/outcomes in adults with TBI.

\section{What was studied in the Cochrane Review?}

The population addressed in this review was adults with TBI. The intervention studied was cognitive rehabilitation referring to the training offered to persons with TBI to address and improve the specific impaired mental functions. The intervention was compared to no intervention (3 studies) or conventional rehabilitation (4 studies). Additionally, the authors compared types of cognitive rehabilitation (cognitive didactic vs. functional experiential, 1 study) as well as intervention settings (in the hospital or at home, 1 study). The outcomes studied included return to work, independence in ADL, community integration, and QoL.

The types and content of cognitive rehabilitation offered to TBI patients varied across studies. Cognitive rehabilitation strategies ranged from interventions for self-awareness, interventions for emotional perception, categorization training, specific therapies targeting emotional regulation, problem solving, and attention training or multi-modal compensatory cognitive training to more comprehensive cognitive rehabilitation strategies (Kumar et al., 2017). Therefore, the data generated by this review relate to the effectiveness of aforementioned cognitive rehabilitation interventions on outcomes specified above and not to the efficacy of cognitive rehabilitation in persons with TBI in a broader context.

None of the studies reported adverse effects of the interventions studied.

\section{Search methodology and up-to-dateness of the Cochrane Review}

Electronic searches were conducted using the Cochrane Central Register of Controlled Trials (CENTRAL) (the Cochrane Library; March 2017, Issue 3), MEDLINE (OvidSP), Embase Classic + Embase (OvidSP), PsycINFO (OvidSP), and clinical trials registries for relevant studies up to 30 March 2017.

\section{What are the main results of the Cochrane Review?}

The review included nine studies.

The review shows that:

- When individuals with TBI who received cognitive rehabilitation were compared to those with no treatment, the intervention did not make any difference regarding return-to-work (very low quality evidence), community integration (low quality evidence), or QoL (low quality evidence).

- When individuals with TBI who received cognitive rehabilitation were compared to those receiving conventional treatment, the intervention did not make any difference regarding return-to-work (low quality evidence), independence in ADL (very low-quality evidence), community integration (low quality evidence), or QoL (moderate-quality evidence).

- When military personnel in active duty who sustained moderate-to-severe TBI received hospital- or home-based cognitive rehabilitation, the groups receiving the intervention in two different settings did not differ regarding return-to work (moderate-quality evidence).

- When military personnel in active duty or adult veterans with moderate-to-severe TBI received two different cognitive rehabilitation strategies, 
those receiving cognitive didactic therapy did not differ from those who received functional experiential therapy regarding return to work (moderate-quality evidence) or independence in ADL (low-quality evidence).

\section{How did the authors conclude on the evidence?}

The authors concluded that there is lack of sufficient good-quality evidence to support the efficacy of cognitive rehabilitation in improving outcomes of return to work, managing ADL independently, and QoL or community integration in adult TBI patients when compared to no treatment or conventional rehabilitation. They found moderate quality evidence indicating similarity of hospital- and home-based cognitive rehabilitation programs in the improvement of return-to-work in a group of military personnel in active duty with moderate to severe TBI. One type of cognitive rehabilitation was found not to be better than the other in military personnel or veterans with TBI for enabling them to return to work based on moderate-quality evidence or independence in ADL based on low quality evidence.

\section{What are the implications of the Cochrane evidence for practice in TBI neurorehabilitation?}

This Cochrane review (Kumar et al., 2017) studied return-to-work as the primary outcome as well as independence in ADL, QoL, and community integration in relation to cognitive rehabilitation in TBI. All of these are very important outcomes relevant to functioning and consequently for TBI neurorecovery rehabilitation. The review provides evidence neither in favor nor against cognitive rehabilitation to improve these outcomes due to insufficient good quality evidence. The quality of evidence in this Cochrane Review ranged from very low quality to moderate quality depending on the outcome assessed. With increasing quality the certainty about the results changes: in case of moderate evidence probably cognitive rehabilitation makes little or no difference to outcomes and the probability that these results will change with other studies is low, while in cases of low and very low quality of evidence the actual results could easily change (Cochrane Norway, 2017; Guyatt et al., 2008). Therefore, a message for rehabilita- tion professionals would be that further studies are warranted to elucidate the effects of cognitive rehabilitation on occupational outcomes and QoL in TBI.

Rehabilitation professionals must conduct good quality research on cognitive rehabilitation relevant to the outcomes of return-to-work, managing ADL, QoL, and participation in the community to create conclusive evidence. To decrease shortcomings in research and to increase the rigor of evidence, there is a need for a common definition and standardization of cognitive rehabilitation and the outcomes (Institute of Medicine, 2011). Furthermore, suggestions by the review authors for future studies include recruitment of study participants with similar severity and duration of TBI as well as factoring the baseline differences by stratification at the recruitment phase, prioritizing outcome measures related to occupational endpoints along with follow-ups longer than a year, studying the effects or possible advantages of intervention in different settings including resourceconstrained settings, and using the TIDieR checklist (Hoffmann, 2014) when reporting to allow the assessment of the homogeneity of similar studies (Kumar et al., 2017).

\section{Acknowledgments}

We thank Cochrane Rehabilitation and Cochrane Work Group for reviewing the contents of the Cochrane Corner.

\section{Conflict of interest}

The author declares no conflicts of interest.

\section{References}

Cicerone, K. D., Langenbahn, D. M., Braden, C., Malec, J. F., Kalmar, K., Fraas, M., ..., \& Ashman, T. (2011). Evidence-based cognitive rehabilitation: Updated review of the literature from 2003 through 2008. Archives of Physical Medicine and Rehabilitation, 92(4), 519-530. doi: 10.1016/j.apmr.2010.11.015

Cochrane Norway (March 27, 2017). How to write a plain language summary of a Cochrane intervention review. Retrieved from: https://www.cochrane.no/sites/cochrane.no/files/public/ uploads/checklist_for_cochrane_pls_28th_feb_2017_0.pdf (Accessed on July 26, 2018)

European Physical and Rehabilitation Medicine Bodies Alliance. (2018). White Book on Physical and Rehabilitation Medicine (PRM) in Europe. Chapter 1. Definitions and concepts of PRM. 
European Journal of Physical and Rehabilitation Medicine, 54(2), 156-165. doi: 10.23736/S1973-9087.18.05144-4

Guyatt, G. H., Oxman, A. D., Vist, G. E., Kunz, R., Falck-Ytter, Y., Alonso-Coello, P., \& Schünemann, H. J.; GRADE Working Group. (2008). GRADE: An emerging consensus on rating quality of evidence and strength of recommendations. BMJ, 336(7650), 924-926. doi: 10.1136/bmj.39489.470347.AD.

Hoffmann, T. C., Glasziou, P. P., Boutron, I., Milne, R., Perera, R., Moher, D., ..., \& Michie S. (2014). Better reporting of interventions: Template for intervention description and replication (TIDieR) checklist and guide. BMJ, 348, g1687. doi: 10.1136/bmj.g1687

Institute of Medicine. (Report Brief released on 10/11/2011). Cognitive rehabilitation therapy for traumatic brain injury:
Evaluating the Evidence. Retrieved from: http://www.natio nalacademies.org/hmd/Reports/2011/Cognitive-Rehabilitati on-Therapy-for-Traumatic-Brain-Injury-Evaluating-the-Evid ence/Report-Brief.aspx (Accessed on July 15, 2018)

Kumar, K. S., Samuelkamaleshkumar, S., Viswanathan, A., \& Macaden, A. S. (2017). Cognitive rehabilitation for adults with traumatic brain injury to improve occupational outcomes. Cochrane Database of Systematic Reviews, 6, CD007935. https://dx.doi.org/10.1002/14651858.CD007935.pub2

WHO. International Classification of Functioning, Disability and Health (ICF). WHO, Geneva, Geneva: World Health Organization, 2001. Retrieved from: http://www.who.int/ classifications/icf/en/ (Accessed on July 11, 2018). 